\title{
A Criterion for the Onset of Chaos in Weakly Dissipative Periodically Driven Systems
}

\author{
S. M. Soskin ${ }^{1}$, R. Mannella ${ }^{2,3}$, A. B. Neiman ${ }^{4}$, A. N. Silchenko ${ }^{4}$, \\ D. G. Luchinsky ${ }^{3}$, and P. V. E. McClintock ${ }^{3}$ \\ ${ }^{1}$ Institute of Semiconductor Physics, Ukainian Academy of Sciences, Kiev, Ukraine \\ ${ }^{2}$ Dipartimento di Fisica, Università di Pisa and INFM UdR Pisa, 56100 Pisa, Italy \\ ${ }^{3}$ Department of Physics, Lancaster University, Lancaster LA1 $4 Y B, U K$ \\ ${ }^{4}$ Department of Physics, Saratov State University, 410026 Saratov, Russia
}

\begin{abstract}
We generalize Chirikov's resonance-overlap criterion for the onset of global chaos in Hamiltonian systems to describe the onset of chaotic attractors in weakly dissipative systems.
\end{abstract}

\section{INTRODUCTION}

The celebrated Chirikov resonance-overlap criterion [1] is based on the idea that, generally, the onset of global chaos in Hamiltonian systems occurs due to strong interactions between resonances of different orders (oscillations of different frequencies calculated in the resonance approximation). The behaviour of weakly dissipative systems is quite similar to that one of non-dissipative ones. Thus it is natural to expect that there should exist a criterion for the onset of chaos, similar to the Chirikov one, applicable in the case of weak dissipation.

To test this hypothesis we study as a simple example the tilted Duffing oscillator (TDO) driven by a periodic force in the presence of weak linear friction. In a certain range of parameters, the TDO belongs to the class of so-called zero-dispersion (ZD) systems [2] whose main characteristic property is an extremum in the dependence of the natural frequency $\omega$ on energy $E$ (Fig. 1). For present purposes, the TDO is particularly convenient for two reasons: (i) it is a simple monostable system, so that periodic driving does not result in complicated behavior related to the saddles of an undriven system; (ii) on account of the minimum in $\omega(E)$, overlap of resonances of different orders may occur at quite small amplitudes of the driving force, so that one may expect that criteria based on the resonance approximation will work better than in those cases that involve large amplitudes.

CP502, Stochastic and Chaotic Dynamics in the Lakes: STOCHAOS,

edited by D. S. Broomhead, E. A. Luchinskaya, P.V. E. McClintock, and T. Mullin

(C) 2000 American Institute of Physics 1-56396-915-7/00/ $\$ 17.00$ 

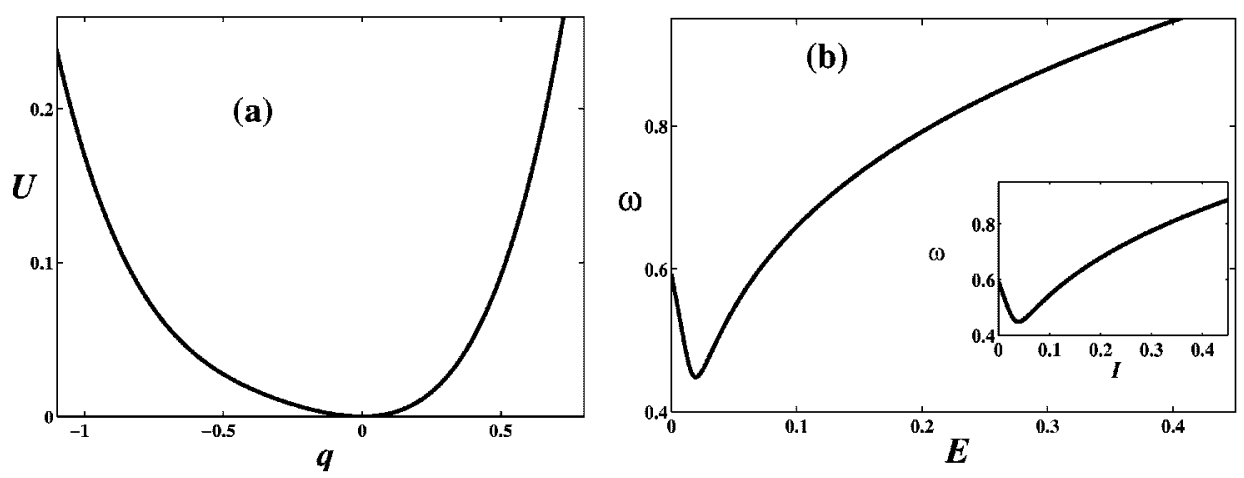

FIGURE 1. (a) The potential (1) at $\omega_{0}=0.5924, \beta=1.026, \gamma=1$; (b) the frequency of eigenoscillation in the potential (1) as a function of energy $E \equiv p^{2} / 2+U(q)$ (or of action $I$, in the inset).

\section{BASIC EQUATIONS}

The coordinate $q$ and momentum $p$ of the system obey the equations

$$
\begin{gathered}
\dot{q}=p, \quad \dot{p}=-\frac{d U}{d q}-\Gamma p+h \cos \left(\omega_{f} t\right), \\
\Gamma \ll \omega_{f}, \quad U(q)=\frac{1}{2} \omega_{0}^{2} q^{2}+\frac{1}{3} \beta q^{3}+\frac{1}{4} \gamma q^{4}, \quad \frac{9}{10}<\frac{\beta^{2}}{\gamma \omega_{0}^{2}}<4 .
\end{gathered}
$$

By transforming to action-angle variables $(I-\psi)$, and omitting non-resonant terms, we obtain the following dynamical equations for the slow variables $I$ and $\tilde{\psi} \equiv$ $n \psi-\omega_{f} t$, describing the motion in the vicinity of $n$-th resonance

$$
\dot{I}=-\frac{\partial \tilde{H}}{\partial \tilde{\psi}}-\Gamma I, \quad \dot{\tilde{\psi}}=\frac{\partial \tilde{H}}{\partial I}, \quad \tilde{H}=\int_{0}^{I} d I\left(n \omega-\omega_{f}\right)-n h q_{n} \cos (\tilde{\psi})
$$

where $q_{n}$ is the coefficient at the $n$-th Fourier component in the expansion of $q(I, \psi)$

$$
q \equiv q(I, \psi)=2 \sum_{n=0}^{\infty} q_{n}(I) \cos (n \psi)
$$

Given the interest in behaviour at relatively small $h$, one may also expect linear response solutions to be relevant:

$$
q_{L R} \approx \frac{h}{\omega_{0}^{2}-\omega_{f}^{2}} \cos \left(\omega_{f} t\right), \quad \omega_{f}-\omega_{0} \gg \Gamma, \omega\left(I=\frac{h^{2} \omega_{0}}{\left(\omega_{0}^{2}-\omega_{f}^{2}\right)^{2}}\right)-\omega_{0}
$$




\section{RESONANCES AND THE NR/ZDNR TRANSITION IN THE REDUCED SYSTEM}

The system (2),(3) is non-chaotic. It possesses attractors most of which correspond approximately to resonant eigenoscillations ${ }^{1}$ i.e. to eigenoscillations at actions $I_{r}$ (or energies $E_{r}$ ) satisfying the resonance condition

$$
n \omega\left(I_{r}\right)=\omega_{f}
$$

That is why we refer to such attractors and their basins as nth-order resonances.

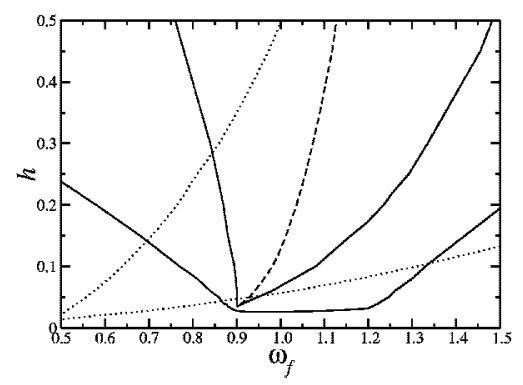

FIGURE 2. The bifurcation diagram for the reduced system (2),(3) at $n=1,2$ for the potential $U(q)$ as in Fig. 1 with $\Gamma=0.05$. The region where two attractors at $n=1$ coexist is limited by dotted lines. There are two (one) attractor(s) corresponding to $n=2$ between inner (inner and outer) full lines. The dashed line marks the NR/ZDNR transition at $n=2$ (see Fig. 3).
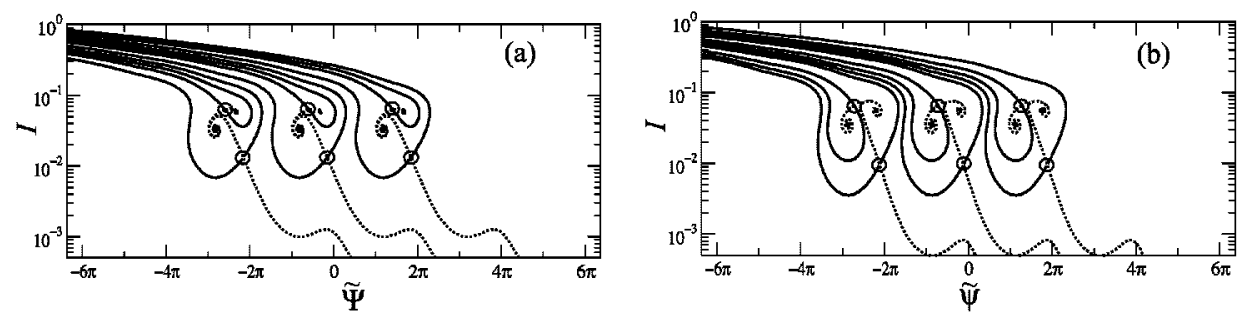

FIGURE 3. Typical evolution of three $2 \pi$ bands of the phase space structure for the reduced system (2),(3) at $n=2$, with the same parameters as in Fig. 2 and $\omega_{f}=0.95$ as $h$ increases from (a) 0.055 to (b) 0.075 ; note that the phase space is periodic in angle, with a period of $2 \pi$ but, in the interest of clarity, we draw only three $2 \pi$ bands. Dots and open circles mark attractors and saddles respectively. Trajectories coming to/from saddles are drawn as full/dotted lines. Trajectories coming to saddles form the boundaries of the basins of attraction.

1) Other attractors, if they exist, correspond at least qualitatively to linear response (4). 
Fig. 2 presents the bifurcation diagrams for $n=1,2$ in the relevant region of the $h-\omega_{f}$ plane. Fig. 3 shows a typical evolution of the phase space structure at $n=2$ as the driving amplitude $h$ increases: the transition from conventional nonlinear resonance (NR) to zero-dispersion nonlinear resonance (ZDNR) occurs [3]. It occurs due to a saddle connection and results in a qualitative change of topology of the basins: in NR (a), the basin of attraction of the smaller-action attractor embraces the basin of the larger-action attractor, while it is other way round in ZDNR (b).

\section{CHAOS IN THE FULL SYSTEM AND AN INTERACTION BETWEEN RESONANCES}

In contrast to the reduced systems (2),(3), the full system (1) has 1.5 degrees of freedom and can be chaotic. Fig. 4 presents the bifurcation diagram for the system (1) together with lines found in the resonance approximation and lines related to the interaction between resonances. There are two different types of chaotic attractor (c.f. Fig.5). The corresponding regions of parameters practically do not overlap. For the sake of brevity, we refer to them as to chaos-1 and chaos-2.

As $h$ increases, the chaotic attractor corresponding to chaos-1 (c.f. Fig. 5(a.7)) is born via a sequence of period-doubling bifurcations from the larger-amplitude period-2 attractor (which corresponds to the larger-action 2nd-order resonance) due to an increasing interaction with the larger-amplitude period-1 attractor (1storder resonance). With further increase of $h$, the chaotic attractor grows and, when $h$ reaches the crisis line, the attractor touches the boundary of attraction of the 1st-order resonance and vanishes discontinuously (Figs.5(a.8,9)).

The chaotic attractor corresponding to chaos-2 (c.f. Fig. 5(b.6)) is born via a sequence of period-doubling bifurcations from the smaller-amplitude period-2 attractor (the smaller-action 2nd-order resonance) due to an increasing interaction with the smaller-amplitude period-1 attractor (linear response). With further increase of $h$, destruction of the chaotic attractor and a reverse sequence of period-doubling bifurcations occur (Figs.5(b.8,9)).

It is seen that the chaos- 1 and chaos- 2 regions can be roughly delineated using simple criteria related to interactions between oscillatory regimes of different frequencies calculated either in the resonance or in linear response approximations.

Chaos-1 is bounded by the line 1-2-3-1. The line 1-2 corresponds to the Chirikovtype overlap in energy between the larger-action 2nd-order resonance and the 1storder nonlinear resonance. The relevance of this line reflects the fact that the origin of chaos-1 lies in a strong interaction between the larger-amplitude period- 1 and period-2 regimes of oscillations (c.f. Figs.5(a.5-7)). The line 1-3 corresponds to the lowest action on the boundary of the 1st-order nonlinear resonance becoming equal to action of the larger-action attractor of the 2nd-order resonance: this line approximately bounds the crisis of the chaotic attractor. The line 2-3 (NR/ZDNR) bounds chaos- 1 region from the right since, in the nearby parameter region, an overlap between the larger-action 2nd-order and 1st-order resonances is possible 


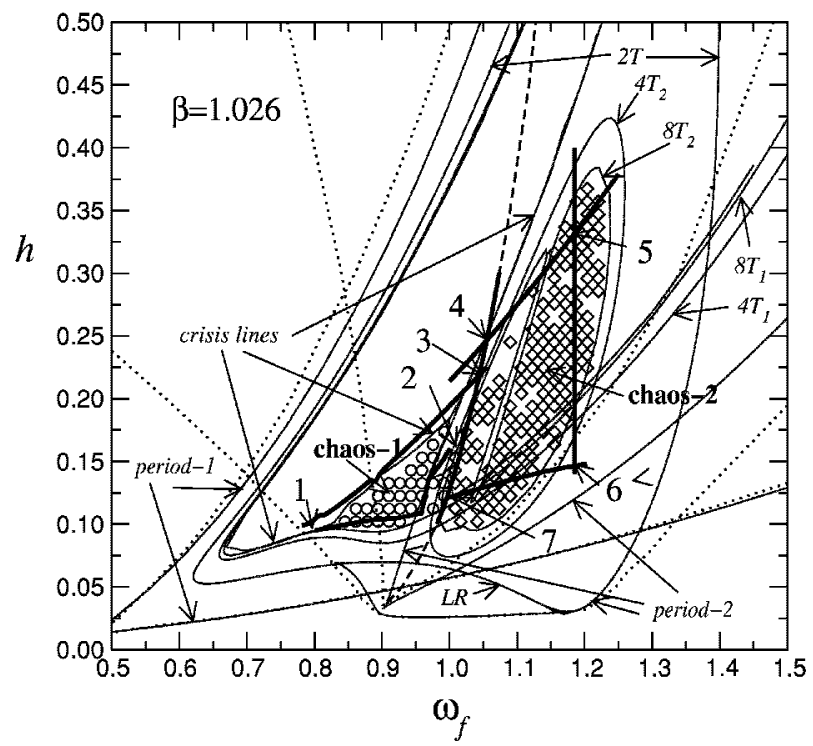

FIGURE 4. The bifurcation diagram for the full system (1) at the same $U(q)$ and $\Gamma$ which are relevant to Fig. 2. Various bifurcations, found from the analysis of Poincare sections, are indicated by thin full lines and by the corresponding labels. Regions where strange attractors exist well match those where positive Lyapunov exponents exist. The latter regions are shaded. To resolve the two different types of chaotic attractors, we use different markers (circles and rhombuses) for shading the corresponding regions; note that there is a slight overlap of the regions (where both types of chaotic attractors coexist). Dotted lines are related to resonances $n=1,2$. Thick full lines are related to the interaction between oscillations of different frequencies, and their intersections are indicated by the arrows and corresponding numbers.

only in the ZDNR stage: in the NR stage, a basin of the larger-action 2nd-order resonance is encompassed by a basin of the smaller-action 2nd-order resonance (see Fig. 3(a) and Fig. 5(a.3)) which prevents an overlap of the former basin with that one of the 1st-order resonance. We note that, as the parameter $\beta$ in $U(q)(1)$ decreases (so that the minimum of $\omega(E)$ becomes shallower and then disappears at $\beta \approx 0.562$ ), an area of the delineated region quickly decreases until it vanishes, which correlates with a disappearance of chaos- 1 at $\beta \approx 0.8$.

Chaos-2 is bounded very roughly by the line $4-5-6-7-4$. Though the delineation is much less accurate than for chaos- 1 it still provides a good guess as to where approximately chaos-2 may be expected and, moreover, the shrinking of the delineated region as $\beta$ decreases describes quite well the shrinking of the chaos- 2 area and its final disappearance at $\beta \approx 0.9$. The line $4-7$, bounding chaos- 2 from the left, is just the NR/ZDNR line: in the region to the right of it, the larger-action 


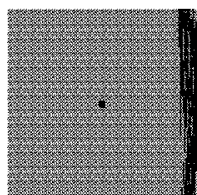

(a.1) $\mathrm{h}=0.02$

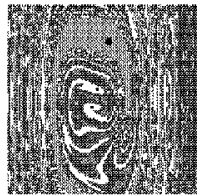

(a.4) $\mathrm{h}=0.061$

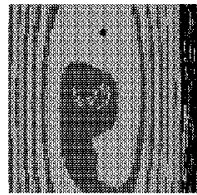

(a.7) $\mathrm{h}=0.113$

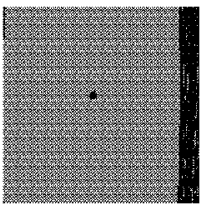

(b.1) $\mathrm{h}=0.02$

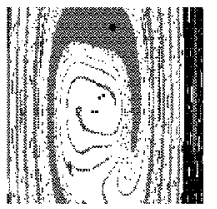

(b.4) $\mathrm{h}=0.08$

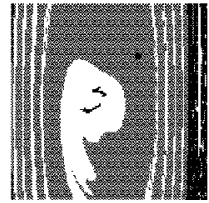

(b.7) $\mathrm{h}=0.190$

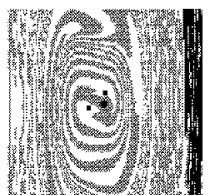

(a.2) $\mathrm{h}=0.031$

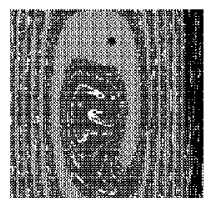

(a.5) $\mathrm{h}=0.075$

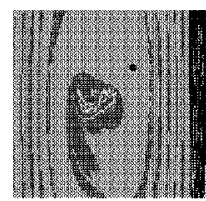

(a.8) $\mathrm{h}=0.150$

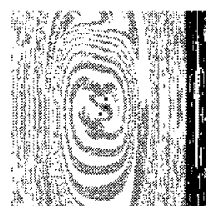

(b.2) $\mathrm{h}=0.04$

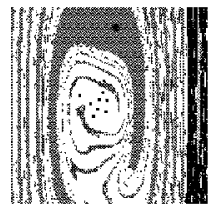

(b.5) $\mathrm{h}=0.086$

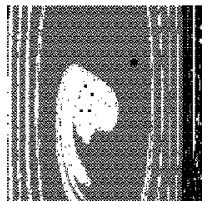

(b.8) $\mathrm{h}=0.215$

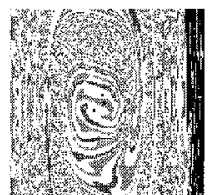

(a.3) $\mathrm{h}=0.05$

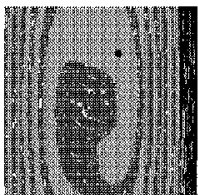

(a.6) $\mathrm{h}=0.10$

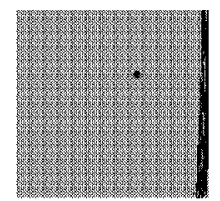

(a.9) $\mathrm{h}=0.20$

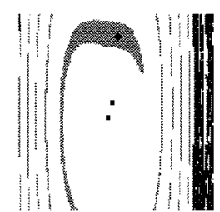

(b.3) $\mathrm{h}=0.068$

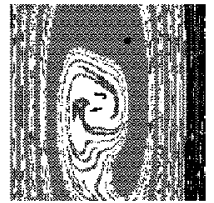

(b.6) $\mathrm{h}=0.110$

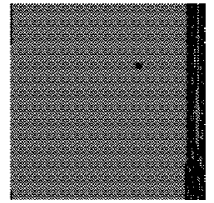

(b.9) $\mathrm{h}=0.227$

FIGURE 5. An evolution of the stroboscopic $(t=0,2 \pi, \ldots)$ Poincare section $p-q$ for: (a) $\omega_{f}=0.95$,(b) $\omega_{f}=1.05$ as $h$ increases: it demonstrates how chaos-1(a) and chaos-2(b) are born. Attractors are indicated by dots, basins of their attraction are shown by different shades. 
2nd-order resonance does not prevent an interaction between the smaller-action 2nd-order resonance and linear response. The lines 6-7 and 4-5 bound (from below and from above respectively) the region where both linear response and the smalleraction 2nd-order resonance are to occur in approximately one and the same energy range: 6-7 and 4-5 correspond to equality of the energy of the attractor of the 2nd-order resonance with the largest and smallest energies respectively of the linear response. The line 5-6, roughly bounding chaos- 2 from the right, is just the line $\omega_{f}=2 \omega_{0}$ : nearly everywhere to the right of it, the smaller-action 2nd-order resonance is suppressed by linear response and, therefore, chaos cannot arise.

Finally, we emphasize that our study is far from being complete: many details are yet to be clarified, the criteria are to be tested on other models. Most importantly, the relationship of the area with chaotic attractors in the weakly dissipative case, to the area with global chaos in the non-dissipative case, needs to be studied.

\section{CONCLUSIONS}

It is evident that chaos may occur in a weakly dissipative periodically driven monostable system (TDO) at very low amplitudes of the driving force. We have demonstrated that its onset/disappearance may readily be interpreted in terms of an interaction between oscillation regimes of different frequencies calculated either in resonance approximation or linear response approximations. We hope that the above criteria may apply generally to all weakly dissipative systems providing rather a simple and convenient tool for the prediction of chaos analogous to that provided by the Chirikov-overlap criterion in non-dissipative systems. To understand the deeper origins of the above criteria will require the involvement of mathematicians.

\section{ACKNOWLEDGMENTS}

The research has been supported by INTAS and by the EPSRC (UK).

\section{REFERENCES}

1. Chirikov, B.V., Phys. Rep. 52, 263-379 (1979).

2. Soskin, S.M., Physica A 155, 401-429 (1989).

3. Luchinsky D.G., McClintock P.V.E., Soskin S.M., and Mannella R., Phys. Rev. Lett. 76, 4453-4457 (1996). 
Copyright $\odot 2003$ EBSCO Publishing 
Copyright of AIP Conference Proceedings is the property of American Institute of Physics and its content may not be copied or emailed to multiple sites or posted to a listserv without the copyright holder's express written permission. However, users may print, download, or email articles for individual use. 